\title{
Predictors of Delay in Seeking Health Care among Myocardial Infarction Patients, Minia District, Egypt
}

\author{
Eman Ramadan Ghazawy, Amany Edward Seedhom, and Eman Mohamed Mahfouz \\ Public Health Department, Faculty of Medicine, Minia University, Minia 61111, Egypt \\ Correspondence should be addressed to Amany Edward Seedhom; amany_medhat@yahoo.com
}

Received 30 September 2015; Accepted 24 November 2015

Academic Editor: Gerardo E. Guillén Nieto

Copyright ( 2015 Eman Ramadan Ghazawy et al. This is an open access article distributed under the Creative Commons Attribution License, which permits unrestricted use, distribution, and reproduction in any medium, provided the original work is properly cited.

\begin{abstract}
Objectives. To determine the barriers that hinder early seeking of medical care among Minia's myocardial infarction patients. Methods. The study was based on individual interviews with 207 men and women with a first confirmed myocardial infarction (MI), admitted to the coronary care units of hospitals in Minia city in the period from April 1 to August 30, 2014. Data was collected via structured questionnaire and patient medical charts. The delay was evaluated by assisting patients to triangulate time of symptom onset and time of professional health care by placing both times in context of daily activities that participants could easily remember. Results. The median (25th, 75th percentiles) delay time was $4(2,10)$ h. Only $32.8 \%$ of patients arrived within 2 hours of symptoms onset. Variables that significantly predicted prehospital delay time were patient's misinterpretation of nature of pain with OR 8.98 (95\% CI) (3.97-20.32), illiteracy 7.98 (2.77-22.95), age (>65) 5.07 (1.57-16.29), and pain resistance behavior 4.61 (2.04-10.41). Conclusions. Interventions to decrease prehospital delay must focus on improving public awareness of acute myocardial infarction symptoms and increasing their knowledge on early treatment benefits.
\end{abstract}

\section{Introduction}

Coronary artery disease (CAD) is the most common cause of morbidity and mortality worldwide [1]. Being a life threatening manifestation of CAD, acute myocardial infarction (AMI) needs prompt recognition and management. Approximately one-third of deaths from AMI occur within few hours of onset of symptoms and usually before the patients reach to hospital [2].

Although AMI is associated with relatively high morbidity and mortality, it is well known that timely reperfusion therapy can result in dramatically improved patient clinical outcomes [3-5].

Delays in seeking medical care are associated with adverse consequences on patients' conditions and medical cost and limit the potential benefits of early interventions $[6,7]$. The quotation "time is muscle" is used to highlight the importance of saving time and starting treatments without delay $[8,9]$.

Early thrombolytic therapy improves perfusion of myocardial ischemic area, limits infarct size, and reduces risk of fatal arrhythmias. It increases survival rate up to $50 \%$ when provided within one hour after symptom onset. However, many patients with AMI do not benefit because of seeking medical care late, as around 50\% seek medical care after 2 hours and more than one-quarter with AMI are referred to the medical center after 6 hours. Factors associated with prolonged prehospital delay in patients with AMI have been the subject of interest in various studies. Many factors such as being old [10], being female [11], having low socioeconomic status, and being Black [12], clinical factors such as a history of hypertension or diabetes, or prior history of angina or previous AMI, and other factors such as consultation with one's spouse, family member, or physician [13] have been associated with longer delay.

However, the majority of these studies were concerned with western populations. Factors associated with prolonged prehospital delay might differ among populations due to diversity in ethnicity, culture, socioeconomic status, health care system organization, and so forth. Recognition of the contributing factors may help to find and develop new interventions to lessen delays and AMI morbidity and mortality rate. 
There is no enough information on the effective factors of delay time in AMI in Minia. Therefore, the aim of the present study was to determine the length of delay and investigate the causes of delay in seeking treatment among Minia patients with AMI.

\section{Methodology}

This cross-sectional study was conducted among all patients with confirmed first acute myocardial infarction (AMI) admitted to the coronary care units of hospitals in Minia city in the period from April 1 to August 30, 2014.

210 patients were interviewed. Patients were pain-free and hemodynamically stable at the time of interview. Individuals diagnosed with the new diagnostic criteria for AMI [14] were eligible for inclusion in the study with no restriction on age. Patients who had a cardiac arrest before admission and those who had cognitive disorders were excluded from the study. Three patients were excluded due to the lack of reliable data.

Total prehospital delay (TPD) was the time (in minutes) from symptom onset to arrival at the emergency department.

The questionnaire contained demographic characteristics of patients, history of medical problems, clinical manifestation of patients at the admission time, sociocultural factors related to delay, and first patient's reactions to MI symptoms. To determine the reliability, a pilot study was conducted among 50 patients and the reliability of this questionnaire was approved using Cronbach's alpha test $(\alpha=0.87)$. It took 15 minutes to fill in the questionnaires through bedside interviews.

The delay was evaluated by assisting patients to triangulate time of symptom onset and time of professional health care by placing both times in context of daily activities that participants could easily remember.

2.1. Statistical Analysis. Data entry and analysis were all done by using software SPSS (Statistical Package for the Social Sciences) version 19. Data were expressed as frequency and percentage, mean \pm standard deviation, or median where appropriate. Differences in the distribution of characteristics of the patients, classified according to the extent of prehospital delay, were examined using the chi-square test for the discrete variables. Logistic regression analysis was utilized to determine which of the sociodemographic, clinical, and behavioral characteristics best distinguished between patients with delay and those without. The probability of less than 0.05 was used as a cutoff point for all significant tests.

\section{Results}

3.1. Baseline and Demographic Characteristics of the Participants. This study included 207 patients diagnosed with AMI. The comparison of prehospital delay times by sociodemographic and clinical characteristics of the patients was presented in Table 1 . The overall median (25th, 75 th percentiles) delay time was $4(2,10) \mathrm{h}$. Of the study group, $32.4 \%$ arrived at hospital within 2 hours after the onset of symptoms.
Among patients, those who delayed seeking medical care for $>2$ hours, it was found that $31.4 \%$ of them were aged patients ( $>65$ years old) and more than half (55\%) were from rural areas compared to $10.5 \%$ and $35.8 \%$ of those who arrived at the hospital within 2 hours, respectively, and these differences were statistically significant ( $p$ value $<0.0001$ and 0.01 ), respectively. Regarding educational and occupational status, it was found that there were statistically significant differences between patients who delayed seeking medical care and those who did not; $46.4 \%$ were illiterates and $47.2 \%$ unemployed, compared to $14.9 \%$ and $23.9 \%$, respectively. Gender and marital status showed no statistically significant differences with prehospital delay.

Concerning clinical characteristics, $20.7 \%$ of patients with prehospital delay $>2$ hours had history of previous angina compared to $38.8 \%$ of those who arrived at the hospital within 2 hours $(p=0.006)$. There was no statistically significant difference between both categories regarding diabetes and hypertension (Table 1).

3.2. Perception of Pain When It Occurs. Regarding the interpretation of the nature of pain, $23.7 \%$ of the participants associated it with a heart problem. The remaining thought of other causes for the pain, interpreting it as a temporary discomfort, a stomach problem, anger, cramps, and more, or even did not imagine what that was.

About $67 \%$ of patients showed pain resistance behavior; we observe that these behaviors are expressed by actions which are attempts to mitigate (e.g., sipping a glass of milk, taking medicine, going to sleep, taking sugar water, and massaging the chest); some patients try to bear and hide the pain (e.g., keeping quiet, not speaking, and not mentioning the pain), and others hope for it to improve and continue activities even with pain (Table 2).

3.3. Causes of Prehospital Delay. Some attitudes were found to contribute to patients' delay in deciding to seek medical attention, 39.3\% of delayed patients did not consider the symptoms to be serious, $10 \%$ found it unpleasant or embarrassing to seek medical help, and $5.7 \%$ did not want to be a burden on anyone.

Other causes related to some contextual factors; as in the patients' opinions, the most common cause of prehospital delay was lack of equipment and proper first line medications, as approximately $25.7 \%$ went to doctors' offices, health centers, and clinics or were seen by a doctor at home, live farther from hospital (13.6\%), and lack suitable transportation (5.7\%) (Table 3).

3.4. Factors Related to Prolonged Prehospital Delay. In multivariate regression analysis (Table 4), it was found that patient's misinterpretation of the nature of pain with OR (95\% CI), 8.98 (3.97-20.32), illiteracy, 7.98 (2.77-22.95), age (>65 years), 5.07 (1.57-16.29), and pain resistance behavior, 4.61 (2.04-10.41), were significant predictors of prolonged prehospital delay. 
TABLE 1: Prehospital delay in relation to baseline and demographic characteristics of the participants.

\begin{tabular}{|c|c|c|c|c|c|c|}
\hline Variables & $\begin{array}{c}\text { All } \\
(n=207)\end{array}$ & $\begin{array}{l}\text { Early arrival } \leq 2 \text { hours } \\
\quad(n=67)=32.3 \%\end{array}$ & $\begin{array}{c}\text { Prehospital } \\
\text { delay }>2 \text { hours } \\
(n=140) 67.7 \%\end{array}$ & $\chi^{2}$ & OR $(95 \% \mathrm{CI})$ & $p$ value \\
\hline \multicolumn{7}{|l|}{ Age } \\
\hline$<50$ years old & $49(23.7)$ & $26(38.8)$ & $23(16.4)$ & \multirow{3}{*}{17.69} & Reference & \multirow{3}{*}{$0.0001^{*}$} \\
\hline $50-65$ years old & $107(52.7)$ & $34(50.7)$ & $73(52.1)$ & & $2.43(1.21-4.85)$ & \\
\hline$>65$ years old & $51(24.6)$ & $7(10.5)$ & $44(31.4)$ & & $7.11(2.68-18.84)$ & \\
\hline \multicolumn{7}{|l|}{ Sex } \\
\hline Male & $155(74.9)$ & $55(82.1)$ & $100(71.4)$ & \multirow{2}{*}{2.73} & Reference & \multirow{2}{*}{0.09} \\
\hline Females & $52(25.1)$ & $12(17.9)$ & $40(28.6)$ & & $1.83(0.88-3.78)$ & \\
\hline \multicolumn{7}{|l|}{ Residence } \\
\hline Urban & $106(51.2)$ & $43(64.2)$ & $63(45)$ & \multirow{2}{*}{6.67} & Reference & \multirow{2}{*}{$0.01^{*}$} \\
\hline Rural & $101(48.8)$ & $24(35.8)$ & $77(55)$ & & $2.19(1.20-3.99)$ & \\
\hline \multicolumn{7}{|l|}{ Marital status } \\
\hline Married & $164(79.3)$ & $57(85.1)$ & $107(76.4)$ & \multirow{2}{*}{2.05} & Reference & \multirow{2}{*}{0.2} \\
\hline Unmarried (widow, single, or divorced) & $43(20.7)$ & $10(14.9)$ & $33(23.6)$ & & $1.75(0.81-3.82)$ & \\
\hline \multicolumn{7}{|l|}{ Educational level } \\
\hline University/above & $58(28.1)$ & $29(43.3)$ & $29(20.7)$ & \multirow{3}{*}{21.66} & Reference & \multirow{3}{*}{$0.0001^{*}$} \\
\hline Below university & $74(35.7)$ & $28(41.8)$ & $46(32.9)$ & & $1.6(0.82-3.29)$ & \\
\hline Illiterate & $75(36.2)$ & $10(14.9)$ & $65(46.4)$ & & $6.50(2.80-15.07)$ & \\
\hline \multicolumn{7}{|l|}{ Occupation } \\
\hline Professional & $43(20.8)$ & $21(31.3)$ & $22(15.7)$ & \multirow{4}{*}{12.51} & Reference & \multirow{4}{*}{$0.006^{*}$} \\
\hline Cleric & $35(16.9)$ & $14(20.9)$ & $21(15)$ & & $1.8(0.79-4.32)$ & \\
\hline Manual working & $47(22.7)$ & $16(23.9)$ & $31(22.1)$ & & $1.4(0.58-3.53)$ & \\
\hline Unemployed & $82(39.6)$ & $16(23.9)$ & $66(47.2)$ & & $3.9(1.75-8.85)$ & \\
\hline \multicolumn{7}{|l|}{ Risk factors } \\
\hline \multicolumn{7}{|l|}{ Hypertension } \\
\hline Yes & $105(50.7)$ & $38(56.7)$ & $67(47.9)$ & \multirow{2}{*}{1.42} & $0.70(0.39-1.25)$ & \multirow{2}{*}{0.2} \\
\hline No & $102(49.3)$ & $29(43.3)$ & $73(52.1)$ & & Reference & \\
\hline \multicolumn{7}{|l|}{ Diabetes } \\
\hline Yes & $98(47.4)$ & $32(47.8)$ & $66(47.1)$ & \multirow{2}{*}{0.007} & $0.97(0.54-1.74)$ & \multirow{2}{*}{0.9} \\
\hline No & $109(52.6)$ & $35(52.2)$ & $74(52.9)$ & & Reference & \\
\hline \multicolumn{7}{|l|}{ Previous angina } \\
\hline Yes & $55(26.6)$ & $26(38.8)$ & $29(20.7)$ & \multirow{2}{*}{7.60} & $0.41(0.21-0.78)$ & \multirow{2}{*}{$0.006^{*}$} \\
\hline No & $152(73.4)$ & $41(61.2)$ & $111(79.3)$ & & Reference & \\
\hline
\end{tabular}

${ }^{*}$ Statistically significant. OR ( $\left.95 \% \mathrm{CI}\right)$ is odds ratio ( $95 \%$ confidence interval).

TABLE 2: Prehospital delay in relation to the acute perception of symptoms.

\begin{tabular}{|c|c|c|c|c|c|c|}
\hline Acute perception of symptoms & $\begin{array}{c}\text { All } \\
(n=207)\end{array}$ & $\begin{array}{l}\text { Early arrival } \leq 2 \text { hours } \\
\qquad(n=67)\end{array}$ & $\begin{array}{c}\text { Prehospital delay }> \\
2 \text { hours } \\
(n=140)\end{array}$ & $\chi^{2}$ & OR $(95 \% \mathrm{CI})$ & $p$ value \\
\hline \multicolumn{7}{|l|}{ Interpretation of the nature of pain } \\
\hline Associate it to heart problem & $49(23.7)$ & $33(49.3)$ & $16(11.4)$ & \multirow{2}{*}{35.8} & Reference & \multirow{2}{*}{$0.0001^{*}$} \\
\hline Misinterpret the nature of pain & $158(76.3)$ & $34(50.7)$ & $124(88.6)$ & & $7.52(3.71-15.62)$ & \\
\hline \multicolumn{7}{|l|}{ Reaction during pain occurrence } \\
\hline Seek medical advice & $69(33.3)$ & $29(43.3)$ & $40(28.6)$ & \multirow{2}{*}{4.4} & Reference & \multirow{2}{*}{$0.03^{*}$} \\
\hline Pain resistance behavior" & $138(66.7)$ & $38(56.7)$ & $100(71.4)$ & & $1.91(1.04-3.50)$ & \\
\hline
\end{tabular}

${ }^{*}$ Statistically significant. OR (95\% CI) is odds ratio (95\% confidence interval)

-Behaviors are expressed by actions which are attempts to mitigate, bear, and hide the pain, hoping for it to improve and to continue activities even with pain. 
TABLE 3: Causes of prehospital delay among study participants.

\begin{tabular}{lcc}
\hline Causes of prehospital delay & No & Percent \\
\hline Causes related to participant attitude & 55 & 39.3 \\
Do not consider the symptoms to be serious & 14 & 10 \\
Find it unpleasant or embarrassing to seek medical help & 8 & 5.7 \\
Do not want to be a burden on anyone & 36 & 25.7 \\
\hline Causes related to surrounding factors & 19 & 13.6 \\
Lack of equipment and proper first line medications & 8 & 5.7 \\
Living in farther distance from hospital & 140 \\
Lack of suitable transportation & 100 \\
\hline Total
\end{tabular}

TABLE 4: Logistic regression for factors influencing prehospital delay for patients with AMI.

\begin{tabular}{|c|c|c|}
\hline Variables & $\begin{array}{c}\text { Odds ratio ( } 95 \% \\
\text { confidence interval) }\end{array}$ & $p$ value \\
\hline $\begin{array}{l}\text { Misinterpret the } \\
\text { nature of pain }\end{array}$ & $8.98(3.97-20.32)$ & $<0.0001$ \\
\hline $\begin{array}{l}\text { Educational level } \\
\text { (illiterate) }\end{array}$ & $7.98(2.77-22.95)$ & $<0.0001$ \\
\hline Age (>65 years old) & $5.07(1.57-16.29)$ & 0.006 \\
\hline $\begin{array}{l}\text { Pain resistance } \\
\text { behavior }\end{array}$ & $4.61(2.04-10.41)$ & $<0.0001$ \\
\hline
\end{tabular}

\section{Discussion}

In this study, the median delay time was 4 hours; this median time is higher than that calculated in a previous study [15] and lower than in another [16]. Two studies showed that a median of prehospital delay in four hospitals in London was 2 hours and in five hospitals in the USA this time was 4.25 hours $[15,16]$.

In the current study it was found that $73.4 \%$ of patients reported that this was the first episode of AMI; this result was consistent with the findings of Mussi et al. [17], who studied the time of decision for seeking medical care and the time of arrival at a health facility for patients with AMI in Salvador and found that vast majority reported that this was the first attack of AMI. This fact may explain why only $23.7 \%$ of the participants associated the pain with a heart problem.

The patients' ability to interpret the symptoms correctly decisively determines their behavior. Our finding confirmed that the majority $(88.6 \%)$ of patients who delayed seeking medical care for $>2$ hours misinterpret the nature of symptoms, and $71.4 \%$ showed pain resistant behaviors. These results were consistent with the findings of Mussi et al. [17], who found that participants who interpreted the pain as cardiovascular in nature took much less time to decide to seek medical care and to reach a health facility and those who expressed pain resistance behaviors took longer time in both to decide to seek medical care and to reach a health facility.

Additionally, the crude OR showed that patients who misinterpreted the nature of symptoms and expressed pain resistance behaviors had about 7- and 2-fold, respectively, increases for delay in seeking help more than those who attributed their symptoms to a cardiac problem and those who did not show pain resistance behaviors (Table 2). Instantly, after adjustment for other factors it showed that both variables were significant predictors in prehospital delay $(p<0.0001)$, with an $\mathrm{OR}=8.98(3.97-20.32)$ and 4.61 (2.04-10.41), respectively. Such results were in accordance with that of Fukuoka et al. (2005) [18] who conducted a study to examine whether culture is associated with a delay in accessing medical care in Japanese patients experiencing symptoms of AMI and found that symptom interpretation on the part of the patients accounted for the largest unique contribution among significant independent variables in prehospital delay time.

A number of sociodemographic, clinical, and behavioral factors have been associated with late presentation at hospital for treatment after the onset of acute coronary symptoms. Based on our results, older people ( $>65$ years old) delayed seeking medical care compared to younger ones. This finding was in agreement with previous published reports declaring that older people experienced longer delay in seeking medical attention compared to younger ones [19-21]. This could be due to older people having limited access to medical care, especially when they live alone. Furthermore, elderly people often have many concomitant diseases that can be regarded as causes of discomfort. Previous investigators have reported conflicting findings about delay times between men and women. Some investigators [22-24] found that women delayed longer than men, while others found no significant differences. We documented no significant differences in delay times between men and women in this current study.

Regarding educational level, illiteracy was found to be a significant predictor for prehospital delay in both univariate and multivariate analysis. This was in accordance with Farshidi et al. [25] who conducted a study to evaluate the factors associated with prehospital delay in patients with AMI in Iran and found that illiterate patients had a higher rate of prehospital delay. Sari and his colleagues (2008) [26], who investigated the factors associated with prolonged prehospital delay in patients with AMI in Turkey, found that total education time of less than nine years was an independent predictor of prolonged prehospital delay OR $=2.27(1.42-$ 3.60). We hypothesized that a high level of education may 
allow better awareness of symptoms and better knowledge of myocardial infarction and therefore reduce symptom-tofirst-medical-contact time, thus explaining our results.

In the current study, we found that employment status was significantly associated with prehospital delay; $47.2 \%$ unemployed patients delayed seeking medical care. This could be explained by the high cost of health care services as the unemployed persons were not covered by governmental health insurance and it could be due to old age because in our sample $41.5 \%$ of the unemployed were $>65$ years old.

In this study causes of prehospital delay were investigated from the patients' point of view. Emotional attitudes were found to be important determinants of patient delay in acute myocardial infarction. The findings of the current study were consistent with Leslie et al. [27] who studied the reasons for the delay and subsequent pattern of accessing care among 313 surviving cases from the Glasgow MONICA (monitoring trends and determinants in cardiovascular disease) coronary event register and found that the most frequently given reason was "thinking that the symptoms would go away" and "not thinking it was serious." And also our findings are in accordance with Kentsch et al. [28] who identified the following independent contributors to a late decision to seek medical help: wanting to wait and see, not taking symptoms seriously, and not wanting to bother anybody.

\section{Limitations of the Study}

Data are dependent on the accuracy and completeness of the individual hospital data abstraction and original chart documentation.

\section{Conclusion and Recommendations}

The process of decision-making regarding whether to seek medical assistance following the onset of chest pain that could be due to a heart attack is multifactorial requiring multifaceted complex interventions. Important elements to address in any intervention seem to be increased awareness about symptoms of AMI, perceived seriousness of the symptoms, and the process of coping with the symptoms adopted. A program of health education about symptoms of AMI and the importance of early seeking care should be conducted through mass media. The authors advise the cardiologists to distribute a brochure about 2 nd prevention to patients after their recovery.

\section{Ethical Approval}

The study protocol had been approved by the standard ethics of Minia University ethical committee for human experimentation.

\section{Consent}

The participants were informed about the purpose of the research and gave written informed consent to participate.

\section{Conflict of Interests}

The authors declare that there is no conflict of interests regarding the publication of this paper.

\section{References}

[1] A. Onat, M. Yazici, I. Sari et al., "The risk factor survey of 2003 in western Turkey indicates trend to declining coronary mortality and urban overall mortality," Türk Kardiyol Dern Arş, vol. 31, no. 12, pp. 762-769, 2003.

[2] L. Chambless, U. Keil, A. Dobson et al., "Population versus clinical view of case fatality from acute coronary heart disease: results from the WHO MONICA Project 1985-1990. Multinational monitoring of trends and determinants in cardiovascular disease," Circulation, vol. 96, no. 11, pp. 3849-3859, 1997.

[3] C. P. Cannon, C. M. Gibson, C. T. Lambrew et al., "Relationship of symptom-onset-to-balloon time and door-to-balloon time with mortality in patients undergoing angioplasty for acute myocardial infarction," The Journal of the American Medical Association, vol. 283, no. 22, pp. 2941-2947, 2000.

[4] G. De Luca, H. Suryapranata, J. P. Ottervanger, and E. M. Antman, "Time delay to treatment and mortality in primary angioplasty for acute myocardial infarction: every minute of delay counts," Circulation, vol. 109, no. 10, pp. 1223-1225, 2004.

[5] D. J. Magid, Y. Wang, J. Herrin et al., "Relationship between time of day, day of week, timeliness of reperfusion, and in-hospital mortality for patients with acute ST-segment elevation myocardial infarction," Journal of the American Medical Association, vol. 294, no. 7, pp. 803-812, 2005.

[6] M. W. Bird, A. G. Woods, and N. A. Warren, "Factors influencing treatment delays for acute myocardial infarction," Critical Care Nursing Quarterly, vol. 32, no. 1, pp. 19-23, 2009.

[7] T. Donyavi, K. H. Naieni, S. Nedjat, M. Vahdaninia, M. Najafi, and A. Montazeri, "Socioeconomic status and mortality after acute myocardial infarction: a study from Iran," International Journal for Equity in Health, vol. 10, no. 1, article 9, 2011.

[8] E. M. Antman, D. T. Anbe, P. W. Armstrong et al., "ACC/AHA guidelines for the management of patients with ST-elevation myocardial infarction; a report of the American College of Cardiology/American Heart Association Task Force on Practice Guidelines (Committee to Revise the 1999 Guidelines for the Management of patients with acute myocardial infarction)," Journal of the American College of Cardiology, vol. 44, no. 3, pp. E1-E211, 2004.

[9] C. M. Gibson, J. A. De Lemos, and E. M. Antman, "Time is muscle in primary PCI: the strength of the evidence grows," European Heart Journal, vol. 25, no. 12, pp. 1001-1002, 2004.

[10] S. McKinley, D. K. Moser, and K. Dracup, “Treatment-seeking behavior for acute myocardial infarction symptoms in North America and Australia," Heart and Lung, vol. 29, no. 4, pp. 237247, 2000.

[11] M. Løvlien, B. Schei, and T. Hole, "Myocardial infarction: psychosocial aspects, gender differences and impact on prehospital delay," Journal of Advanced Nursing, vol. 63, no. 2, pp. 148-154, 2008.

[12] J. J. Zerwic, C. J. Ryan, H. A. DeVon, and M. J. Drell, “Treatment seeking for acute myocardial infarction symptoms: differences in delay across sex and race," Nursing Research, vol. 52, no. 3, pp. 159-167, 2003. 
[13] J. R. Quinn, "Delay in seeking care for symptoms of acute myocardial infarction: applying a theoretical model," Research in Nursing and Health, vol. 28, no. 4, pp. 283-294, 2005.

[14] K. Thygesen, J. S. Alpert, and H. D. White, "Universal definition of myocardial infarction," European Heart Journal, vol. 28, no. 20, pp. 2525-2538, 2007.

[15] L. Perkins-Porras, D. L. Whitehead, P. C. Strike, and A. Steptoe, "Pre-hospital delay in patients with acute coronary syndrome: factors associated with patient decision time and home-tohospital delay," European Journal of Cardiovascular Nursing, vol. 8, no. 1, pp. 26-33, 2009.

[16] A. D. Banks and K. Dracup, "Factors associated with prolonged prehospital delay of African Americans with acute myocardial infarction," American Journal of Critical Care, vol. 15, no. 2, pp. 149-157, 2006.

[17] F. C. Mussi, A. S. Mendes, T. L. de Queiroz, A. L. Costa, Á. Pereira, and B. Caramelli, "Pre-hospital delay in acute myocardial infarction: judgement of symptoms and resistance to pain," Revista da Associação Médica Brasileira, vol. 60, no. 1, pp. 63-69, 2014.

[18] Y. Fukuoka, K. Dracup, S. H. Rankin et al., "Prehospital delay and independent/interdependent construal of self among Japanese patients with acute myocardial infarction," Social Science and Medicine, vol. 60, no. 9, pp. 2025-2034, 2005.

[19] R. J. Goldberg, J. H. Gurwitz, and J. M. Gore, "Duration of, and temporal trends (1994-1997) in, prehospital delay in patients with acute myocardial infarction: the second National Registry of Myocardial Infarction," Archives of Internal Medicine, vol. 159, no. 18, pp. 2141-2147, 1999.

[20] R. J. Goldberg, J. Yarzebski, D. Lessard, and J. M. Gore, "Decadelong trends and factors associated with time to hospital presentation in patients with acute myocardial infarction: the Worcester Heart Attack study," Archives of Internal Medicine, vol. 160, no. 21, pp. 3217-3223, 2000.

[21] M. M. Ottesen, U. Dixen, C. Torp-Pedersen, and L. Køber, "Prehospital delay in acute coronary syndrome-an analysis of the components of delay," International Journal of Cardiology, vol. 96, no. 1, pp. 97-103, 2004.

[22] H. Meischke, M. P. Larsen, and M. S. Eisenberg, "Gender differences in reported symptoms for acute myocardial infarction: impact on prehospital delay time interval," The American Journal of Emergency Medicine, vol. 16, no. 4, pp. 363-366, 1998.

[23] S. E. Sheifer, S. S. Rathore, B. J. Gersh et al., "Time to presentation with acute myocardial infarction in the elderly: associations with race, sex, and socioeconomic characteristics," Circulation, vol. 102, no. 14, pp. 1651-1656, 2000.

[24] S. A. Murphy, C. Chen, C. P. Cannon, E. M. Antman, and C. M. Gibson, "Impact of gender on angiographic and clinical outcomes after fibrinolytic therapy in acute myocardial infarction," American Journal of Cardiology, vol. 90, no. 7, pp. 766-770, 2002.

[25] H. Farshidi, S. Rahimi, A. Abdi, S. Salehi, and A. Madani, "Factors associated with pre-hospital delay in patients with acute myocardial infarction," Iranian Red Crescent Medical Journal, vol. 15, no. 4, pp. 312-316, 2013.

[26] I. Sari, Z. Acar, O. Özer et al., "Factors associated with prolonged prehospital delay in patients with acute myocardial infarction," Archives of the Turkish Society of Cardiology, vol. 36, no. 3, pp. 156-162, 2008.

[27] W. S. Leslie, A. Urie, J. Hooper, and C. E. Morrison, "Delay in calling for help during myocardial infarction: reasons for the delay and subsequent pattern of accessing care," Heart, vol. 84, no. 2, pp. 137-141, 2000.
[28] M. Kentsch, U. Rodemerk, G. Müller-Esch et al., "Emotional attitudes toward symptoms and inadequate coping strategies are major determinants of patient delay in acute myocardial infarction," Zeitschrift für Kardiologie, vol. 91, no. 2, pp. 147-155, 2002. 


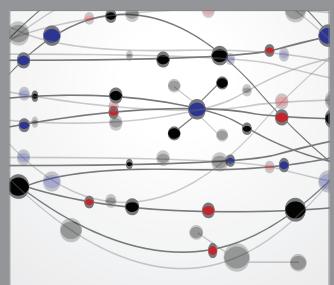

The Scientific World Journal
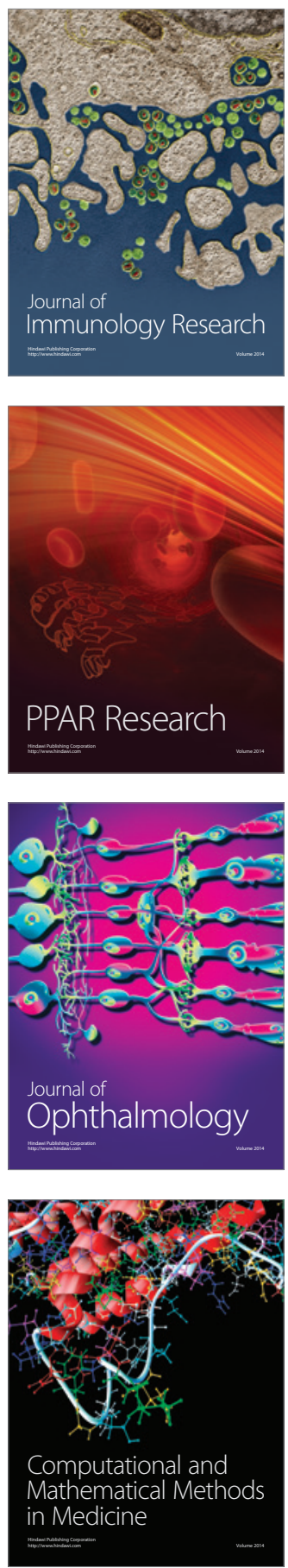

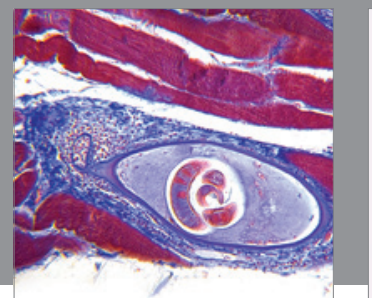

Gastroenterology

Research and Practice
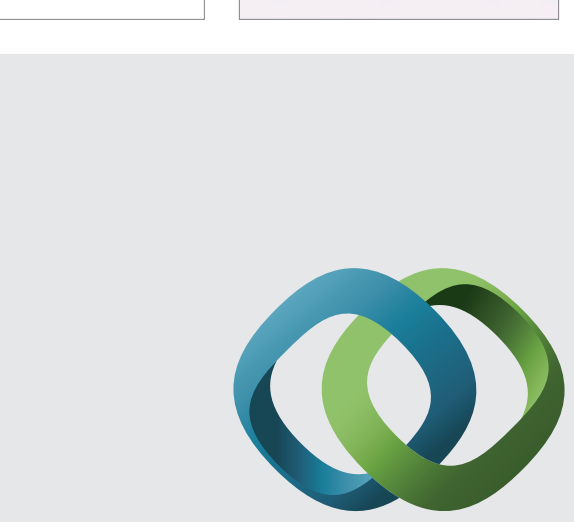

\section{Hindawi}

Submit your manuscripts at

http://www.hindawi.com
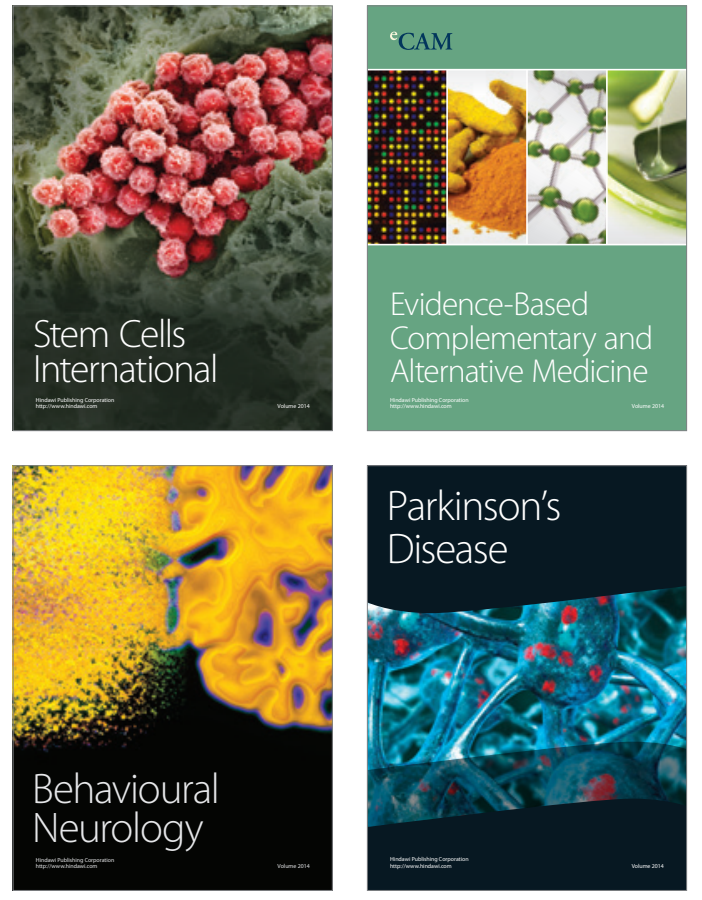
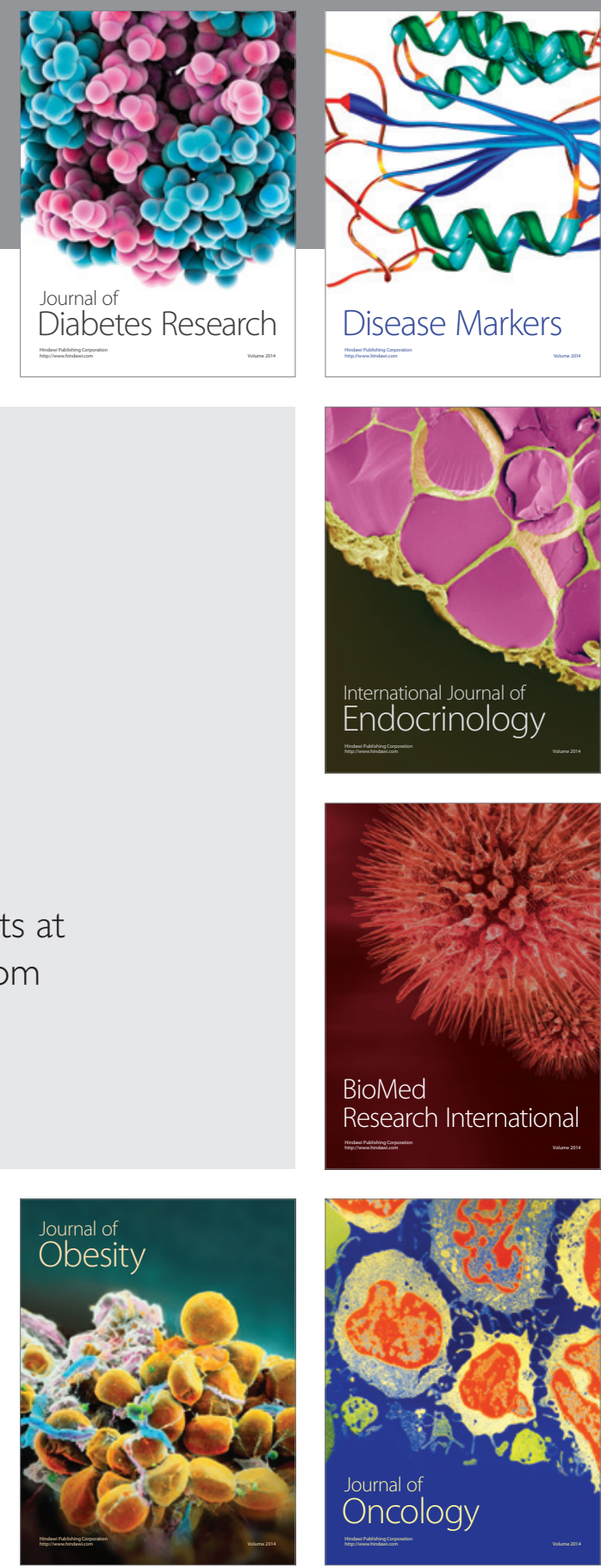

Disease Markers
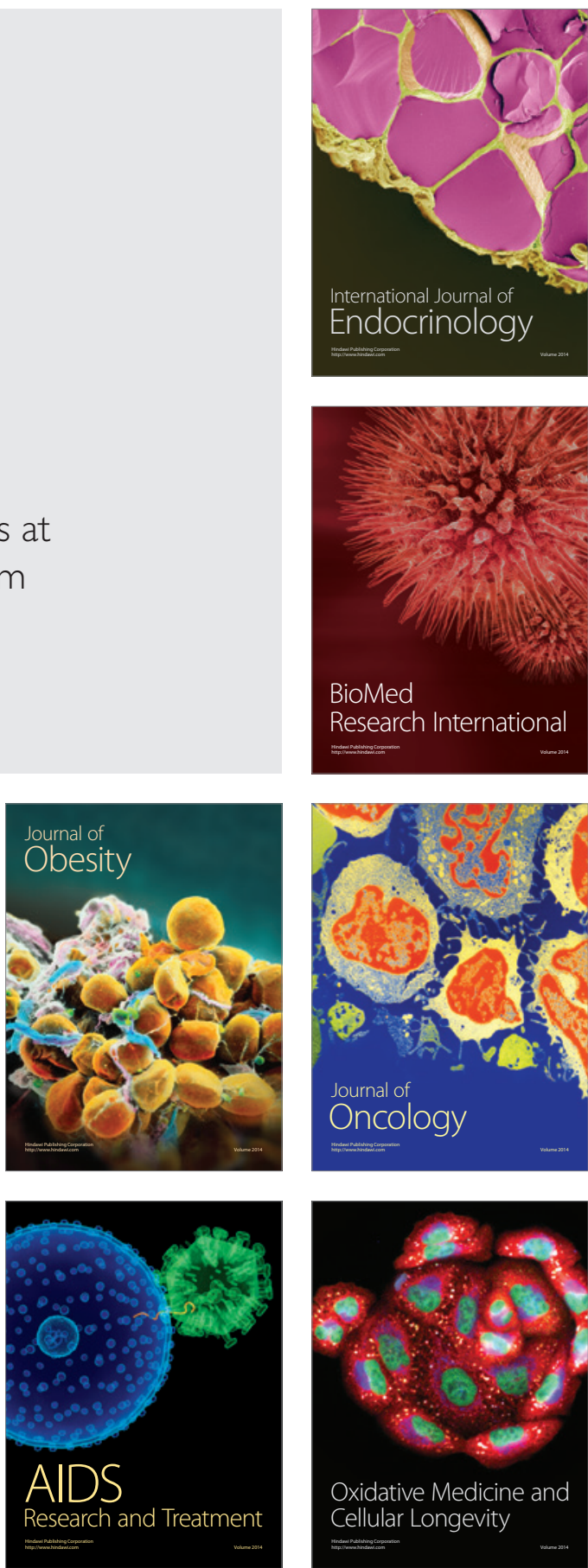